\title{
Vortex buoyancy in superfluid and superconducting neutron stars
}

\author{
V. A. Dommes, M. E. Gusakov \\ Ioffe Institute, Polytekhnicheskaya 26, 194021 St. Petersburg, Russia
}

\begin{abstract}
Buoyancy of proton vortices is considered as one of the important mechanisms of magnetic field expulsion from the superconducting interiors of neutron stars. Here we show that the generally accepted expression for the buoyancy force is not correct and should be modified. The correct expression is derived for both neutron and proton vortices. It is argued that this force is already contained in the coarse-grained hydrodynamics of Bekarevich \& Khalatnikov and its various multifluid extensions, but is absent in the hydrodynamics of Hall. Some potentially interesting buoyancy-related effects are briefly discussed.
\end{abstract}

Key words: stars: neutron - stars: magnetic fields - stars: interiors - hydrodynamics

\section{INTRODUCTION}

According to theoretical calculations (e.g., Lombardo \& Schulze 2001; Page et al. 2013), protons in the neutron star cores condense into a superconducting state at temperatures below $T \sim 10^{8}-10^{10} \mathrm{~K}$. If they form a type-II superconductor, the magnetic flux penetrates the core in the form of quantized fluxtubes (also called Abrikosov vortices or, simply, proton vortices), each carrying a single flux quantum $\phi_{0} \equiv \pi \hbar c / e_{\mathrm{p}}$, where $\hbar, c$, and $e_{\mathrm{p}}$ are the Planck constant, speed of light, and proton electric charge, respectively.

Since the magnetic field in the neutron star core is locked to proton vortices, the problem of its evolution reduces to that of vortex motion. This motion is, in turn, determined by the balance of forces acting on vortices. One of these forces is the so called buoyancy force, introduced into the neutron-star literature by Muslimov \& Tsygan (1985) and used subsequently in many works studying the magnetic field expulsion from the superconducting interiors of neutron stars (e.g. Harvey et al. 1986; Jones 1987; Srinivasan et al. 1990; Chau et al. 1992; Ding et al. 1993; Chau 1997; Jahan-Miri 2000, 2002; Konenkov \& Geppert 2000, 2001; Elfritz et al. 2016). Note that this force should, in principle, act on both proton and neutron (FeynmanOnsager) vortices.

The buoyancy force was derived in Muslimov \& Tsygan (1985) from the purely hydrodynamic consideration. One may ask whether it is legitimate to apply such an approach on microscopic length-scales comparable to the radius of the vortex core. Another issue is related to the fact that the buoyancy force is always added in the equation of vortex motion as some 'external' force. This implies that it should not be contained, for example, in the smooth-averaged hy- drodynamics of Bekarevich \& Khalatnikov (1961) (and its multifluid extensions). But is it true?

We address these issues in the present note. The work is organized as follows. Section 2 contains a brief discussion of the vortex structure, together with the Muslimov \& Tsygan derivation of the buoyancy force, and alternative derivation leading to a different result. The new expression for the buoyancy force is then compared to the results of microscopic calculations based on the solution to the conservative Ginzburg-Landau (Gross-Pitaevsky) equation. Section 3 clarifies whether the buoyancy effects are already present in the hydrodynamics of Bekarevich \& Khalatnikov (1961). Section 4 considers a few applications of our results. Finally, we sum up in section 5. For simplicity, we neglect the relativistic and entrainment effects in this note. They are thoroughly discussed in Gusakov \& Dommes (2016).

\section{BUOYANCY FORCE ON A VORTEX}

\section{$2.1 \quad$ Vortex structure}

Consider first a proton vortex. It consists of a nonsuperconducting core with the radius of the order of the proton coherence length $\xi_{\mathrm{p}} \equiv \hbar p_{\mathrm{Fp}} /\left(\pi m_{\mathrm{p}}^{*} \Delta_{\mathrm{p}}\right)$,

$\xi_{\mathrm{p}} \approx 1.7 \times 10^{-12} \mathrm{~cm}\left(\frac{n_{\mathrm{p}}}{0.18 n_{0}}\right)^{1 / 3}\left(\frac{m_{\mathrm{p}}}{m_{\mathrm{p}}^{*}}\right)\left(\frac{0.76 \mathrm{MeV}}{\Delta_{\mathrm{p}}}\right)$,

surrounded by superconducting currents with the velocity field (e.g., De Gennes 1999; Landau \& Lifshitz 1980)

$\boldsymbol{v}(\boldsymbol{r})=\frac{e_{\mathrm{p}} \phi_{0}}{2 \pi m_{\mathrm{p}} c \delta_{\mathrm{p}}} K_{1}\left(\frac{r}{\delta_{\mathrm{p}}}\right) \boldsymbol{e}_{\varphi}$. 
Here $p_{\mathrm{Fp}}, n_{\mathrm{p}}, m_{\mathrm{p}}, m_{\mathrm{p}}^{*}, \Delta_{\mathrm{p}}$ are the proton Fermi momentum, number density, mass, effective mass, and energy gap, respectively; $n_{0}=0.16 \mathrm{fm}^{-3}$ is the nuclear matter density; $r$ is the distance to the vortex core; $\boldsymbol{e}_{\varphi}$ is the unit vector in the azimuthal direction ${ }^{1}$; and $K_{i}(x)$ is the MacDonald function. Finally, $\delta_{\mathrm{p}} \equiv\left[m_{\mathrm{p}}^{2} c^{2} /\left(4 \pi e_{\mathrm{p}}^{2} \rho_{\mathrm{sp}}\right)\right]^{1 / 2}$ is the London penetration depth for protons,

$\delta_{\mathrm{p}} \approx 4.24 \times 10^{-12} \mathrm{~cm}\left(\frac{0.18 n_{0}}{n_{\mathrm{sp}}}\right)^{1 / 2}$,

where $\rho_{\mathrm{sp}}$ and $n_{\mathrm{sp}}=\rho_{\mathrm{sp}} / m_{\mathrm{p}}$ are, respectively, the 'superconducting' proton mass density and number density. At $r \ll \delta_{\mathrm{p}}$ the velocity $v(r) \approx \kappa_{\mathrm{p}} /(2 \pi r)\left[\kappa_{\mathrm{p}} \equiv \pi \hbar / m_{\mathrm{p}}\right.$ is the circulation quantum], while at $r \gg \delta_{\mathrm{p}}$ it decays exponentially, $v(r) \propto \exp \left(-r / \delta_{\mathrm{p}}\right)$. The proton supercurrent produces a magnetic field,

$\boldsymbol{B}(r)=\frac{\phi_{0}}{2 \pi \delta_{\mathrm{p}}^{2}} K_{0}\left(\frac{r}{\delta_{\mathrm{p}}}\right) \boldsymbol{\nu}$,

where $\boldsymbol{\nu} \equiv \boldsymbol{e}_{z}$ is the unit vector in the vortex direction (along $z)$. The vortex energy per unit length, $\hat{E}_{\mathrm{Vp}}$, is given by the formula (Landau \& Lifshitz 1980)

$\hat{E}_{\mathrm{Vp}}=\int\left[\rho_{\mathrm{sp}} \frac{v^{2}(r)}{2}+\frac{B^{2}(r)}{8 \pi}\right] r \mathrm{~d} r \mathrm{~d} \varphi \approx \rho_{\mathrm{sp}} \frac{\kappa_{\mathrm{p}}^{2}}{4 \pi} \ln \frac{\delta_{\mathrm{p}}}{\xi_{\mathrm{p}}}$.

To allow for the entrainment effect, $\rho_{\mathrm{sp}}$ in equation (5) should be replaced with the element $\rho_{\text {pp }}$ of the entrainment matrix $\rho_{i k}$ (Mendell 1991).

Similar formulas can also be written for vortices in an uncharged superfluids, e.g., for neutron vortex ${ }^{2}$. Its velocity field is $\boldsymbol{v}(\boldsymbol{r})=\kappa_{\mathrm{n}} /(2 \pi r) \boldsymbol{e}_{\varphi}$, and energy per unit length

$\hat{E}_{\mathrm{Vn}} \approx \rho_{\mathrm{sn}} \frac{\kappa_{\mathrm{n}}^{2}}{4 \pi} \ln \frac{b_{\mathrm{n}}}{\xi_{\mathrm{n}}}$,

where $\kappa_{\mathrm{n}} \equiv \pi \hbar / m_{\mathrm{n}} ; \xi_{\mathrm{n}}$ is the neutron coherence length, given by the formula similar to equation (1); and $b_{\mathrm{n}}$ is some 'external' radius of the order of the typical length-scale of the problem (e.g., intervortex spacing or radius of a vessel). To account for the entrainment, $\rho_{\mathrm{sn}}$ in equation (6) should be replaced with the combination $\left(\rho_{\mathrm{nn}} \rho_{\mathrm{pp}}-\rho_{\mathrm{np}}^{2}\right) / \rho_{\mathrm{pp}}$ (Mendell 1991; Glampedakis et al. 2011).

Note that in inhomogeneous medium (when $\rho_{\text {sn }}$ slowly varies in space) the neutron vortex velocity differs from $\boldsymbol{v}(\boldsymbol{r})=\kappa_{\mathrm{n}} /(2 \pi r) \boldsymbol{e}_{\varphi}$ (see, e.g., Rubinstein \& Pismen 1994; Sheehy \& Radzihovsky 2004), but the vortex energy $\hat{E}_{\mathrm{Vn}}$ still has the same form (6) up to the terms quadratic in $\left|\nabla \rho_{\text {sn }}\right|$. The same, of course, applies to proton vortices.

\subsection{Buoyancy force: standard derivation}

The concept of the buoyancy force on a vortex was introduced by Muslimov \& Tsygan (1985) (see also Harvey et al. 1986) who considered proton fluxtubes in superconducting interiors of neutron stars. These authors noted that in the vicinity of the vortex core the fluid pressure (and, therefore,

1 We use the cylindrical coordinate system $(r, \varphi, z)$ with the axis $z$ directed along the vortex line.

2 In this paper we assume, for simplicity, that neutrons pair in the spin-singlet $\left({ }^{1} \mathrm{~S}_{0}\right)$ state. density) decreases because of local magnetic field and supercurrents associated with the vortex. The pressure drop $\Delta P(r)$ at a distance $r$ from the vortex line is given by

$\Delta P(r)=\rho_{\mathrm{sp}} \frac{v^{2}(r)}{2}+\frac{B^{2}(r)}{8 \pi}$.

Therefore, the buoyancy force per unit length $\boldsymbol{f}_{B}$, arising due to the density drop $\Delta \rho(r)$, equals

$\boldsymbol{f}_{B}=-\boldsymbol{g} \int_{\xi_{\mathrm{p}}}^{\infty} \Delta \rho(r) r \mathrm{~d} r \mathrm{~d} \varphi=-\hat{E}_{\mathrm{Vp}} \frac{\boldsymbol{g}}{c_{\mathrm{s}}^{2}}$,

where $\boldsymbol{g}$ is the local gravitational acceleration. Here we used the relation $\Delta \rho=c_{s}^{-2} \Delta P$, where $c_{\mathrm{s}}^{2} \equiv \mathrm{d} P / \mathrm{d} \rho$ is the squared speed of sound. Using the hydrostatic equilibrium condition $\rho \boldsymbol{g}=\nabla P$, one can rewrite the buoyancy force as

$\boldsymbol{f}_{B}=-\hat{E}_{\mathrm{Vp}} \frac{\boldsymbol{g}}{c_{\mathrm{s}}^{2}}=-\hat{E}_{\mathrm{Vp}} \frac{\nabla \rho}{\rho}$.

The proposed simple derivation predicts that there is a nonzero force acting on a vortex in an external gravitational field. One may see that this force is derived from the purely 'hydrodynamic' arguments. However, the validity of such a hydrodynamic approach is questionable at microscopic length-scales (e.g., use of the notion of pressure in the very vicinity of the vortex core looks doubtful). Below we demonstrate that equation (9) is correct only in one special case and, generally, it should be replaced by a different formula.

\subsection{Alternative derivation and different result}

The force acting on a vortex in inhomogeneous medium can also be derived from simple energetic arguments. Assume first that the vortex is directed along the axis $z$, while the background superfluid density $\rho_{\mathrm{sp}}$ depends on $x$ only [here and below we use the standard Cartesian coordinates $(x, y, z)]$. Now, if we shift the vortex from $x$ to $x^{\prime}=x+\delta x$, its energy (5) will change according to

$\hat{E}_{\mathrm{Vp}}\left(x^{\prime}\right)-\hat{E}_{\mathrm{Vp}}(x) \approx\left[\rho_{\mathrm{sp}}\left(x^{\prime}\right)-\rho_{\mathrm{sp}}(x)\right] \frac{\kappa_{\mathrm{p}}^{2}}{4 \pi} \ln \frac{\delta_{\mathrm{p}}}{\xi_{\mathrm{p}}}$,

so that the buoyancy force, given by the (minus) vortex energy gradient, will be $\boldsymbol{f}_{B}=-\boldsymbol{\nabla} \hat{E}_{\mathrm{Vp}}(\boldsymbol{r})$. In the more general case, when $\nabla \rho_{\text {sp }}$ is not necessarily directed along $x$, one has

$\boldsymbol{f}_{B}=-\nabla_{\perp} \hat{E}_{\mathrm{Vp}}(\boldsymbol{r})=-\hat{E}_{\mathrm{Vp}} \frac{\nabla_{\perp} \rho_{\mathrm{sp}}}{\rho_{\mathrm{sp}}}$

where $\nabla_{\perp} \equiv \boldsymbol{\nabla}-\boldsymbol{\nu}(\boldsymbol{\nu} \boldsymbol{\nabla})$ is the component of gradient perpendicular to the vortex line direction $\boldsymbol{\nu}$. The appearance of $\nabla_{\perp}$ instead of $\boldsymbol{\nabla}$ in this formula reflects the fact that the translation of the vortex along the axis $z$ does not change its energy. Of course, similar formula (with index $p$ replaced by $n$ ) also applies to neutron (Feynman-Onsager) vortices (Sheehy \& Radzihovsky 2004; see also section 2.4).

Generally, the formula (11) differs from the 'classical' result (9). They are equivalent only for a one-component liquid in the hydrostatic equilibrium and at zero temperature $(T=0)$, when $\rho_{\mathrm{sp}}=\rho$.

\subsection{Comparison with the microscopic theory}

It is interesting to confront our formula (11) with the results of microscopic theory. For example, Rubinstein \& Pismen 
(1994) analysed, within the conservative Ginzburg-Landau (Gross-Pitaevsky) equation, the motion of a neutral (e.g., neutron) vortex in the system at $T=0$ with a slightly inhomogeneous background superfluid density $\rho_{\mathrm{sn}}(x, y)$. They considered a situation in which the vortex is straight and directed along the axis $z$. In such formulation the problem is two-dimensional - the vortex moves in the $x y$ plane. Using the method of matched asymptotic expansion, Rubinstein \& Pismen (1994) found the following analytical expression for the vortex velocity $\boldsymbol{V}_{\mathrm{Ln}}$ :

$\boldsymbol{V}_{\mathrm{Ln}}=\boldsymbol{V}_{\mathrm{sn}}-\frac{\kappa_{\mathrm{n}}}{4 \pi} \boldsymbol{\nu} \times \frac{\nabla \rho_{\mathrm{sn}}}{\rho_{\mathrm{sn}}} \ln \frac{R_{\mathrm{n}}}{\xi_{\mathrm{n}}}$.

Here $\boldsymbol{V}_{\mathrm{sn}}$ is the background superfluid velocity and $R_{\mathrm{n}}$ is a typical length-scale of superfluid density variation,

$R_{\mathrm{n}}=2 a_{1} e^{1 / 2-C} / \sqrt{\left(\frac{\nabla \rho_{\mathrm{sn}}}{2 \rho_{\mathrm{sn}}}\right)^{2}-\frac{1}{2} \nabla^{2} \ln \rho_{\mathrm{sn}}}$

where $C \approx 0.577$ is the Euler constant and $\ln a_{1} \approx 0.405$.

One can try to derive an equation similar to (12) from the formula (11) (see also Sheehy \& Radzihovsky 2004). The only forces acting on a vortex in the conservative $2 \mathrm{D}$ problem considered by Rubinstein \& Pismen (1994) are the Magnus force, $\boldsymbol{f}_{\mathrm{M}}=\rho_{\mathrm{sn}} \kappa_{\mathrm{n}} \boldsymbol{\nu} \times\left(\boldsymbol{V}_{\mathrm{Ln}}-\boldsymbol{V}_{\mathrm{sn}}\right)$, and the buoyancy force, $\boldsymbol{f}_{\mathrm{B}}$. Neglecting vortex inertia, one should have $\boldsymbol{f}_{\mathrm{B}}+\boldsymbol{f}_{\mathrm{M}}=0$, which gives ${ }^{3}$,

$\boldsymbol{V}_{\mathrm{Ln}}=\boldsymbol{V}_{\mathrm{sn}}-\frac{\hat{E}_{\mathrm{Vn}}}{\rho_{\mathrm{sn}} \kappa_{\mathrm{n}}} \boldsymbol{\nu} \times \frac{\boldsymbol{\nabla} \rho_{\mathrm{sn}}}{\rho_{\mathrm{sn}}}=\boldsymbol{V}_{\mathrm{sn}}-\frac{\kappa_{\mathrm{n}}}{4 \pi} \boldsymbol{\nu} \times \frac{\boldsymbol{\nabla} \rho_{\mathrm{sn}}}{\rho_{\mathrm{sn}}} \ln \frac{b_{\mathrm{n}}}{\xi_{\mathrm{n}}}$.

This equation has exactly the same structure as (12) provided that we identify $b_{\mathrm{n}}=R_{\mathrm{n}}$, which is natural, since this is the only 'external' length-scale in the problem. It follows from (14) that in the absence of background superfluid velocity $\left(\boldsymbol{V}_{\mathrm{sn}}=0\right)$ vortex moves along the surface $\rho_{\mathrm{sn}}=$ const, i.e. its energy is conserved.

\section{IS THERE A BUOYANCY FORCE IN SUPERFLUID HYDRODYNAMICS?}

In the context of neutron stars the vortex buoyancy (in the form 8) was routinely introduced as some external force (but see Jones 2006) in order to explain expulsion of the magnetic field (confined to Abrikosov vortices) from the superconducting interiors of neutron stars. Here we shall demonstrate that this force is absent in the often used version (Hall 1960) of superfluid hydrodynamics of Hall \& Vinen (1956) (see also a monograph by Donnelly 2005) but is contained in the hydrodynamics of Bekarevich \& Khalatnikov (1961) (and its various extensions). Thus, introduction of this force 'by hands' may lead to double-counting. Note that, the fact that the buoyancy is already incorporated in the hydrodynamic equations of Bekarevich \& Khalatnikov (1961), has not been well recognized in the literature (see, e.g., p. 94 of the authoritative review by Sonin 1987, where this force is described as 'external').

3 Note that $\boldsymbol{V}_{\text {Ln }}$ is defined up to an arbitrary term parallel to the vortex line. Such term does not affect the vortex dynamics (see, e.g., Khalatnikov 2000) and is chosen here such that $\boldsymbol{V}_{\mathrm{Ln}} \boldsymbol{\nu}=$ $\boldsymbol{V}_{\mathrm{sn}} \boldsymbol{\nu}$.
Consider a neutral superfluid (e.g., neutron liquid) containing vortices moving with some local velocity $\boldsymbol{V}_{\text {Ln }}$. For simplicity, we shall be interested in the so called weak-drag limit, when the mutual friction forces can be neglected (note that, effectively, Rubinstein \& Pismen 1994 also worked in that limit, see section 2.4). In this approximation the hydrodynamics of Bekarevich \& Khalatnikov (1961) gives the following expression for $\boldsymbol{V}_{\mathrm{Ln}}$ (see equation 16.45 in the monograph by Khalatnikov 2000 with $\beta=\beta^{\prime}=0^{4}$ ),

$\boldsymbol{V}_{\mathrm{Ln}}^{(\mathrm{BK})}=\boldsymbol{V}_{\mathrm{sn}}+\frac{1}{\rho_{\mathrm{sn}}} \operatorname{curl} \lambda_{\mathrm{n}} \boldsymbol{\nu}=\boldsymbol{V}_{\mathrm{sn}}+\frac{1}{\rho_{\mathrm{sn}}} \operatorname{curl}\left(\frac{\hat{E}_{\mathrm{Vn}}}{\kappa_{\mathrm{n}}} \boldsymbol{\nu}\right)$

$=\boldsymbol{V}_{\mathrm{sn}}-\frac{\hat{E}_{\mathrm{Vn}}}{\kappa_{\mathrm{n}} \rho_{\mathrm{sn}}} \boldsymbol{\nu} \times \frac{\nabla \rho_{\mathrm{sn}}}{\rho_{\mathrm{sn}}}+\frac{\hat{E}_{\mathrm{Vn}}}{\kappa_{\mathrm{n}} \rho_{\mathrm{sn}}} \operatorname{curl} \boldsymbol{\nu}$,

where $\lambda_{\mathrm{n}} \equiv \hat{E}_{\mathrm{Vn}} / \kappa_{\mathrm{n}}$ and in the second line we make use of the fact that $\hat{E}_{\mathrm{Vn}}$ is approximately proportional to $\rho_{\mathrm{sn}}$ [see equation (6)]. In turn, the hydrodynamics of Hall (1960) predicts that in the weak-drag limit $\boldsymbol{V}_{\text {Ln }}$ equals

$\boldsymbol{V}_{\mathrm{Ln}}^{(\mathrm{H})}=\boldsymbol{V}_{\mathrm{sn}}+\frac{\hat{E}_{\mathrm{Vn}}}{\kappa_{\mathrm{n}} \rho_{\mathrm{sn}}} \operatorname{curl} \boldsymbol{\nu}$.

Let us compare equations (15), (16), and equation (14) from the previous section. First of all, the last terms in the r.h.s. of equations (15) and (16) coincide and appear due to the local vortex curvature (and related tension), which generates small contribution to $\boldsymbol{V}_{\mathrm{Ln}}$, making it a bit different from the average 'transport' velocity $\boldsymbol{V}_{\text {sn }}$ (see Donnelly 2005 for details; note, however, that the corresponding discussion in that reference contains a number of misprints). Because this term vanishes for straight vortices, it does not appear in equation (14).

Next, the second term in the r.h.s. of equation (15) indicates that the difference between the vortex velocity $\boldsymbol{V}_{\mathrm{Ln}}$ and the transport velocity $\boldsymbol{V}_{\text {sn }}$ is driven not only by vortex curvature, but also by inhomogeneity of the background, $\nabla \rho_{\text {sn }}$. It exactly coincides with the similar 'buoyancy' term in equation (14), but is absent in the Hall's hydrodynamics [see equation (16)].

We come to conclusion that the superfluid hydrodynamics of Bekarevich \& Khalatnikov (1961) implicitly contains all buoyancy effects, whereas one has to include the buoyancy force 'by hands' if one prefers to use the superfluid hydrodynamics of Hall (1960).

It remains to note that the buoyancy effects are automatically included in both non-relativistic and relativistic extensions of Bekarevich and Khalatnikov hydrodynamics, describing superfluid/superconducting mixtures (see, e.g., Mendell \& Lindblom 1991; Mendell 1991; Sedrakian \& Sedrakian 1995; Glampedakis et al. 2011; Gusakov 2016; Gusakov \& Dommes 2016).

\section{SOME APPLICATIONS}

\subsection{Vortices in a rotating neutral superfluid}

Let us discuss, within the hydrodynamics of Bekarevich \& Khalatnikov (1961), the equilibrium configuration of a rotating neutral superfluid with non-zero

4 Note a misprint in this equation: the second term in its r.h.s. should have ' + ' sign in front of $1 / \rho_{\mathrm{s}} \operatorname{curl} \lambda \boldsymbol{\nu}$. 
$\nabla \rho_{\text {sn }}$. For simplicity, we restrict ourselves to the cylindrical geometry (e.g., cylindrical vessel rotating at a frequency $\boldsymbol{\Omega}$ around its symmetry axis), and consider the case of a one-component liquid at $T=0$ (hence $\rho_{\text {sn }}$ equals the total mass density $\left.\rho_{\mathrm{n}}\right)$. Generalization of these results to finite temperatures is straightforward and does not change our main conclusions.

The superfluid is governed by the 'superfluid' and continuity equations (see equations 16.24 and 16.40 in Bekarevich \& Khalatnikov 1961), which can be rewritten in the frame, rotating with the vessel, as

$$
\begin{aligned}
& \frac{\partial \tilde{\boldsymbol{V}}_{\mathrm{sn}}}{\partial t}+2\left[\boldsymbol{\Omega} \times \tilde{\boldsymbol{V}}_{\mathrm{sn}}\right]+\left(\tilde{\boldsymbol{V}}_{\mathrm{sn}} \boldsymbol{\nabla}\right) \tilde{\boldsymbol{V}}_{\mathrm{sn}}+\boldsymbol{\nabla}(\breve{\mu}+\phi) \\
& =-\frac{1}{\rho_{\mathrm{sn}}}\left[\operatorname{curl} \tilde{\boldsymbol{V}}_{\mathrm{sn}} \times \operatorname{curl}\left(\lambda_{\mathrm{n}} \boldsymbol{\nu}\right)\right]-\frac{1}{\rho_{\mathrm{sn}}}\left[2 \boldsymbol{\Omega} \times \operatorname{curl}\left(\lambda_{\mathrm{n}} \boldsymbol{\nu}\right)\right] \\
& \frac{\partial \rho_{\mathrm{sn}}}{\partial t}+\operatorname{div}\left(\rho_{\mathrm{sn}} \tilde{\boldsymbol{V}}_{\mathrm{sn}}\right)=0,
\end{aligned}
$$

where $\tilde{\boldsymbol{V}}_{\mathrm{sn}}=\boldsymbol{V}_{\mathrm{sn}}-[\boldsymbol{\Omega} \times \boldsymbol{r}]$ is measured in the rotating frame; $\breve{\mu}$ is the chemical potential per unit mass; and $\phi$ is the sum of centrifugal potential plus potential of some external force producing the density gradient (in neutron stars it is the gravitational potential). In the absence of density gradients, when the right-hand side of equation (17) vanishes ${ }^{5}$, one has $\tilde{\boldsymbol{V}}_{\mathrm{sn}}=0$ in equilibrium, that is superfluid mimics solid-body rotation. However, when the density gradients are allowed for, the solution to equations (17) and (18) describes differential rotation,

$\tilde{\boldsymbol{V}}_{\mathrm{sn}}=\frac{\lambda_{\mathrm{n}}}{\rho_{\mathrm{sn}}} \nu \times \frac{\nabla \rho_{\mathrm{sn}}}{\rho_{\mathrm{sn}}}$.

At the same time, using (15) one obtains that vortices move with the vessel (and with the normal component if $T \neq 0$ ): $\boldsymbol{V}_{\text {Ln }}=[\boldsymbol{\Omega} \times \boldsymbol{r}]$. For neutron stars the typical precession frequency of vortices with respect to the superfluid component, $\omega_{\mathrm{P}} \equiv\left|\tilde{\boldsymbol{V}}_{\mathrm{sn}}\right| / r=\kappa_{\mathrm{n}} /(4 \pi r) \ln \left(b_{\mathrm{n}} / \xi_{\mathrm{n}}\right)\left|\boldsymbol{\nabla}\left(\ln \rho_{\mathrm{sn}}\right)\right|$, is extremely small, $\omega_{\mathrm{P}} \sim 10^{-15} \mathrm{~s}^{-1}$, but it is much larger (and is observed) in Bose-Einstein condensates (Anderson et al. 2000). It is interesting that a similar formula for $\omega_{P}$ has been obtained in Sheehy \& Radzihovsky (2004) by different means.

\subsection{Magnetic field evolution in superconducting neutron-star cores}

If protons in a neutron star core form a type-II superconductor, then almost all magnetic field is confined to proton vortices (e.g., Glampedakis et al. 2011). Therefore, evolution of the magnetic induction $\boldsymbol{B}$ in the superconducting region is governed by the equation (e.g., Konenkov \& Geppert 2001),

$\frac{\partial \boldsymbol{B}}{\partial t}=\operatorname{curl}\left[\boldsymbol{V}_{\mathrm{Lp}} \times \boldsymbol{B}\right]$,

which describes transport of $\boldsymbol{B}$ with the velocity $\boldsymbol{V}_{\mathrm{Lp}}$ of proton vortices. The latter is given by the expression

$\boldsymbol{V}_{\mathrm{Lp}}=\boldsymbol{V}_{\mathrm{norm}}-\alpha_{\mathrm{p}} \boldsymbol{W}_{\mathrm{p}}-\beta_{\mathrm{p}} \boldsymbol{\nu} \times \boldsymbol{W}_{\mathrm{p}}$,

5 Note that $\operatorname{curl} \boldsymbol{\nu}=0$ because $\boldsymbol{\nu}$ is collinear with $\boldsymbol{\Omega}$. where $\boldsymbol{W}_{\mathrm{p}}$ can be presented as a sum of two terms related, respectively, to buoyancy and vortex tension,

$\boldsymbol{W}_{\mathrm{p}}=\operatorname{curl}\left(\lambda_{\mathrm{p}} \boldsymbol{\nu}\right)=-\frac{\hat{E}_{\mathrm{Vp}}}{\kappa_{\mathrm{p}}} \boldsymbol{\nu} \times \frac{\boldsymbol{\nabla} \rho_{\mathrm{sp}}}{\rho_{\mathrm{sp}}}+\frac{\hat{E}_{\mathrm{Vp}}}{\kappa_{\mathrm{p}}} \operatorname{curl} \boldsymbol{\nu}$.

In equations (21) and (22) $\lambda_{\mathrm{p}}=\hat{E}_{\mathrm{Vp}} / \kappa_{\mathrm{p}} ; \boldsymbol{\nu}=\boldsymbol{B} / B ; \alpha_{\mathrm{p}}$ and $\beta_{\mathrm{p}}$ are the (poorly known) mutual friction coefficients. These equations have been recently derived in Gusakov \& Dommes (2016) under assumption that all the normal (non-superfluid and non-superconducting) liquid components move with one and the same 'normal' velocity $\boldsymbol{V}_{\text {norm }}{ }^{6}$. This assumption can be relaxed (Gusakov \& Dommes, in preparation), which modifies $\boldsymbol{V}_{\mathrm{Lp}}$ by adding a diffusion-induced term to $\boldsymbol{W}_{\mathrm{p}}$.

It is instructive to estimate typical time-scales appearing in the evolution equation (20). Following Graber et al. (2015), one gets

$$
\begin{aligned}
& \tau_{\text {cons }}=\frac{\kappa_{\mathrm{p}} L^{2}}{\left|\alpha_{\mathrm{p}}\right| \hat{E}_{\mathrm{Vp}}}=\frac{4 \pi L^{2}}{\left|\alpha_{\mathrm{p}}\right| \rho_{\mathrm{sp}} \kappa_{\mathrm{p}}}\left[\ln \left(\frac{\delta_{\mathrm{p}}}{\xi_{\mathrm{p}}}\right)\right]^{-1}, \\
& \tau_{\text {diss }}=\frac{\kappa_{\mathrm{p}} L^{2}}{\beta_{\mathrm{p}} \hat{E}_{\mathrm{Vp}}}=\frac{4 \pi L^{2}}{\beta_{\mathrm{p}} \rho_{\mathrm{sp}} \kappa_{\mathrm{p}}}\left[\ln \left(\frac{\delta_{\mathrm{p}}}{\xi_{\mathrm{p}}}\right)\right]^{-1} .
\end{aligned}
$$

Here $L$ is the characteristic length-scale of the problem, characterizing variation of the magnetic induction $\boldsymbol{B}$, or superfluid density $\rho_{\mathrm{sp}}$, or vortex direction $\nu$. Note that, near the normal-superconducting boundary, the ratio $\nabla \rho_{\mathrm{sp}} / \rho_{\mathrm{sp}}$ [see the 'buoyancy' term in equation (22)] can be very large, since there $\rho_{\mathrm{sp}} \rightarrow 0$, while $\nabla \rho_{\mathrm{sp}}$ is finite. Also, $\nabla \rho_{\mathrm{sp}}$ can be large (i.e., $L$ is small) near the crust-core interface and, generally, in the vicinity of any place in the core where the superfluid proton density varies sharply. ${ }^{7}$

The time-scales (23) and (24) are called, respectively, 'conservative' and 'dissipative' because it can be shown (e.g., Mendell \& Lindblom 1991; Gusakov \& Dommes 2016) that the terms $\propto \beta_{\mathrm{p}}$ create entropy in the system, while those $\propto \alpha_{\mathrm{p}}$ do not. The mutual friction coefficients $\alpha_{\mathrm{p}}$ and $\beta_{\mathrm{p}}$ are rather uncertain, especially at temperatures comparable to the nucleon critical temperatures, and there are no agreement in the literature on their actual values (e.g., Alpar et al. 1984; Jones 2006; Andersson et al. 2006; Jones 2009). At $T=0$ these coefficients can be expressed through the drag coefficient $\mathcal{R}_{\mathrm{p}} \sim 2 \times 10^{-4}$ (Graber et al. 2015) as

$\alpha_{\mathrm{p}}=-\frac{1}{\rho_{\mathrm{sp}}} \frac{1}{1+\mathcal{R}_{\mathrm{p}}^{2}}, \quad \beta_{\mathrm{p}}=\frac{1}{\rho_{\mathrm{sp}}} \frac{\mathcal{R}_{\mathrm{p}}}{1+\mathcal{R}_{\mathrm{p}}^{2}}$.

Adopting these values, one obtains, to leading order in $\mathcal{R}_{\mathrm{p}}$, $\tau_{\text {cons }} \approx 2 \times 10^{8} L_{6}^{2} \mathrm{yr}, \quad \tau_{\text {diss }} \approx 2 \times 10^{12} L_{6}^{2}\left(\frac{\mathcal{R}_{\mathrm{p}}}{10^{-4}}\right)^{-1} \mathrm{yr}$,

where $L_{6}=L /\left(10^{6} \mathrm{~cm}\right)$. Clearly, these estimates should be taken with caution. Still, they indicate that the time-scale $\tau_{\text {cons }}$ can be comparable to the typical time-scale of magnetic field evolution in pulsars.

Note that $\tau_{\text {cons }}$ is smaller than the corresponding estimate in Graber et al. (2015) by 7 orders of magnitude, which is a consequence of a bit different evolution equation used

6 Graber et al. (2015) proposed a magnetic field evolution equation which is different from (21); we discuss it below.

7 We thank A.I. Chugunov for pointing out to us this possibility. 
by these authors. The evolution equation of Graber et al. (2015) (their equation 67) reads ${ }^{8}$

$\frac{\partial \boldsymbol{B}}{\partial t}=\operatorname{curl}\left[\left(\boldsymbol{V}_{\mathrm{Lp}}-\underline{\underline{\hat{E}_{\mathrm{Vp}}}} \underline{\underline{\rho_{\mathrm{p}} \kappa_{\mathrm{p}}}} \operatorname{curl} \boldsymbol{\nu}\right) \times \boldsymbol{B}\right]$,

where the expression for the vortex velocity,

$\boldsymbol{V}_{\mathrm{Lp}}=\boldsymbol{V}_{\mathrm{norm}}+\frac{\hat{E}_{\mathrm{Vp}}}{\rho_{\mathrm{sp}} \kappa_{\mathrm{p}}\left(1+\mathcal{R}_{\mathrm{p}}^{2}\right)}\left(\operatorname{curl} \boldsymbol{\nu}-\mathcal{R}_{\mathrm{p}} \boldsymbol{\nu} \times \operatorname{curl} \boldsymbol{\nu}\right)$,

coincides with equation (21) if $\alpha_{\mathrm{p}}$ and $\beta_{\mathrm{p}}$ are given by equation (25) and, additionally, $\nabla \rho_{\mathrm{sp}}=0$ (which means that the authors ignore the buoyancy force). Equation (27) is rather puzzling: it states that the magnetic field is transported with the velocity that differs from the vortex velocity $\boldsymbol{V}_{\mathrm{Lp}}$, in contradiction to the (explicitly made) assumption that all the magnetic field is stored in vortices. Note that this equation has been used subsequently by Elfritz et al. (2016) for detailed modelling of the magnetic field evolution in neutron stars (see equation 16 in that reference). These authors also accounted for the buoyancy force, but in the incorrect form (9).

Now the reason for the seven-order difference in the estimates for $\tau_{\text {cons }}$ can be easily pinpointed. To this aim we expand the second term in equation (28) in the Taylor series in small parameter $\mathcal{R}_{\mathrm{p}}$. The leading-order contribution in that expansion cancels out the underlined term in equation (27). As a result, $\tau_{\text {cons }}$ in Graber et al. (2015) is larger than our estimate $(23)$ by a factor $\sim \mathcal{R}_{\mathrm{p}}^{-2} \sim 10^{7}$.

\section{SUMMARY}

Our results can be summarized as follows:

(i) The standard expression (9) for the buoyancy force acting on a vortex is generally incorrect and should be replaced with equation (11). Essentially, one has to replace in equation (9) $\boldsymbol{g} / c_{\mathrm{s}}^{2}=\nabla \rho / \rho$ with $\nabla_{\perp} \rho_{\mathrm{sp}} / \rho_{\mathrm{sp}}$ (for proton vortices), or with $\nabla_{\perp} \rho_{\mathrm{sn}} / \rho_{\mathrm{sn}}$ (for neutron vortices). In some regions of a neutron star this can significantly increase the buoyancy force (and may even reverse its direction). In particular, buoyancy effects can be important near the boundary of superconducting region, where $\rho_{\mathrm{sp}}$ varies sharply.

(ii) The proposed buoyancy force derivation is supported by microscopic calculations within the conservative Ginzburg-Landau (Gross-Pitaevsky) equation (Rubinstein \& Pismen 1994; see also Sheehy \& Radzihovsky 2004).

(iii) The same buoyancy force is already implicitly accounted for in the superfluid hydrodynamics of Bekarevich \& Khalatnikov (1961) and its extensions to superfluid/superconducting mixtures (Mendell \& Lindblom 1991; Mendell 1991; Sedrakian \& Sedrakian 1995; Glampedakis et al. 2011; Gusakov \& Dommes 2016). To the best of our knowledge, the corresponding terms in the equations describing superfluids have not been interpreted as 'buoyancy-related' (see, e.g., p. 94 of the

8 It also immediately follows from equations (160) and (162) of Glampedakis et al. (2011). review by Sonin 1987, where the buoyancy force is explicitly considered as 'external').

(iv) However, this force does not appear in superfluid hydrodynamics of Hall (1960) (see also Donnelly 2005), which is equivalent to that of Bekarevich \& Khalatnikov in (almost) all other aspects.

(v) We briefly discussed a number of applications in which the buoyancy force may lead to noticeable effects. These include hydrostatic equilibrium of neutral rotating superfluids, as well as the magnetic field evolution in superconducting neutron stars. Our estimates indicate that the magnetic field can evolve on a much shorter timescale than it was assumed by Graber et al. (2015) (and, subsequently, by Elfritz et al. 2016), who used a different (and incorrect, as we argue in Section 4) magnetic field evolution equation.

\section{ACKNOWLEDGEMENTS}

We are very grateful to Elena Kantor, Andrey Chugunov, Kostas Glampedakis, and D.A. Shalybkov for valuable discussions and comments. This study was supported by the Russian Science Foundation (grant number 14-12-00316).

\section{REFERENCES}

Alpar M. A., Langer S. A., Sauls J. A., 1984, ApJ, 282, 533

Anderson B. P., Haljan P. C., Wieman C. E., Cornell E. A., 2000, Physical Review Letters, 85, 2857

Andersson N., Sidery T., Comer G. L., 2006, MNRAS, 368, 162

Bekarevich I. L., Khalatnikov I. M., 1961, JETP, 13, 643

Chau H. F., 1997, ApJ, 479, 886

Chau H. F., Cheng K. S., Ding K. Y., 1992, ApJ, 399, 213

De Gennes P., 1999, Superconductivity Of Metals And Alloys. Advanced Books Classics Series, Westview Press

Ding K. Y., Cheng K. S., Chau H. F., 1993, ApJ, 408, 167

Donnelly R. J., 2005, Quantized Vortices in Helium II

Elfritz J. G., Pons J. A., Rea N., Glampedakis K., Viganò D., 2016, MNRAS, 456, 4461

Glampedakis K., Andersson N., Samuelsson L., 2011, MNRAS, 410, 805

Graber V., Andersson N., Glampedakis K., Lander S. K., 2015, MNRAS, 453, 671

Gusakov M. E., 2016, Phys. Rev. D, 93, 064033

Gusakov M. E., Dommes V. A., 2016, Phys. Rev. D, 94, 083006

Hall H. E., 1960, Advances in Physics, 9, 89

Hall H. E., Vinen W. F., 1956 , Proceedings of the Royal Society of London Series A, 238,215

Harvey J. A., Ruderman M. A., Shaham J., 1986, Phys. Rev. D, 33,2084

Jahan-Miri M., 2000, ApJ, 532, 514

Jahan-Miri M., 2002, Phys. Rev. B, 65, 184522

Jones P. B., 1987, MNRAS, 228, 513

Jones P. B., 2006, MNRAS, 365, 339

Jones P. B., 2009, MNRAS, 397, 1027

Khalatnikov I. M., 2000, An introduction to the theory of superfluidity. Westview Press, New York

Konenkov D., Geppert U., 2000, MNRAS, 313, 66

Konenkov D., Geppert U., 2001, MNRAS, 325, 426

Landau L. D., Lifshitz E. M., 1980, Statistical physics. Pt.2. Pergamon Press, Oxford

Lombardo U., Schulze H.-J., 2001, in Blaschke D., Glendenning N. K., Sedrakian A., eds, Lecture Notes in Physics, Berlin 
Springer Verlag Vol. 578, Physics of Neutron Star Interiors. p. 30

Mendell G., 1991, ApJ, 380, 515

Mendell G., Lindblom L., 1991, Annals of Physics, 205, 110

Muslimov A. G., Tsygan A. I., 1985, Ap\&SS, 115, 43

Page D., Lattimer J. M., Prakash M., Steiner A. W., 2013, preprint, (arXiv:1302.6626)

Rubinstein B. Y., Pismen L. M., 1994, Physica D Nonlinear Phenomena, 78, 1

Sedrakian A. D., Sedrakian D. M., 1995, ApJ, 447, 305

Sheehy D. E., Radzihovsky L., 2004, Phys. Rev. A, 70, 063620

Sonin E. B., 1987, Reviews of Modern Physics, 59, 87

Srinivasan G., Bhattacharya D., Muslimov A. G., Tsygan A. J., 1990, Current Science, 59, 31 